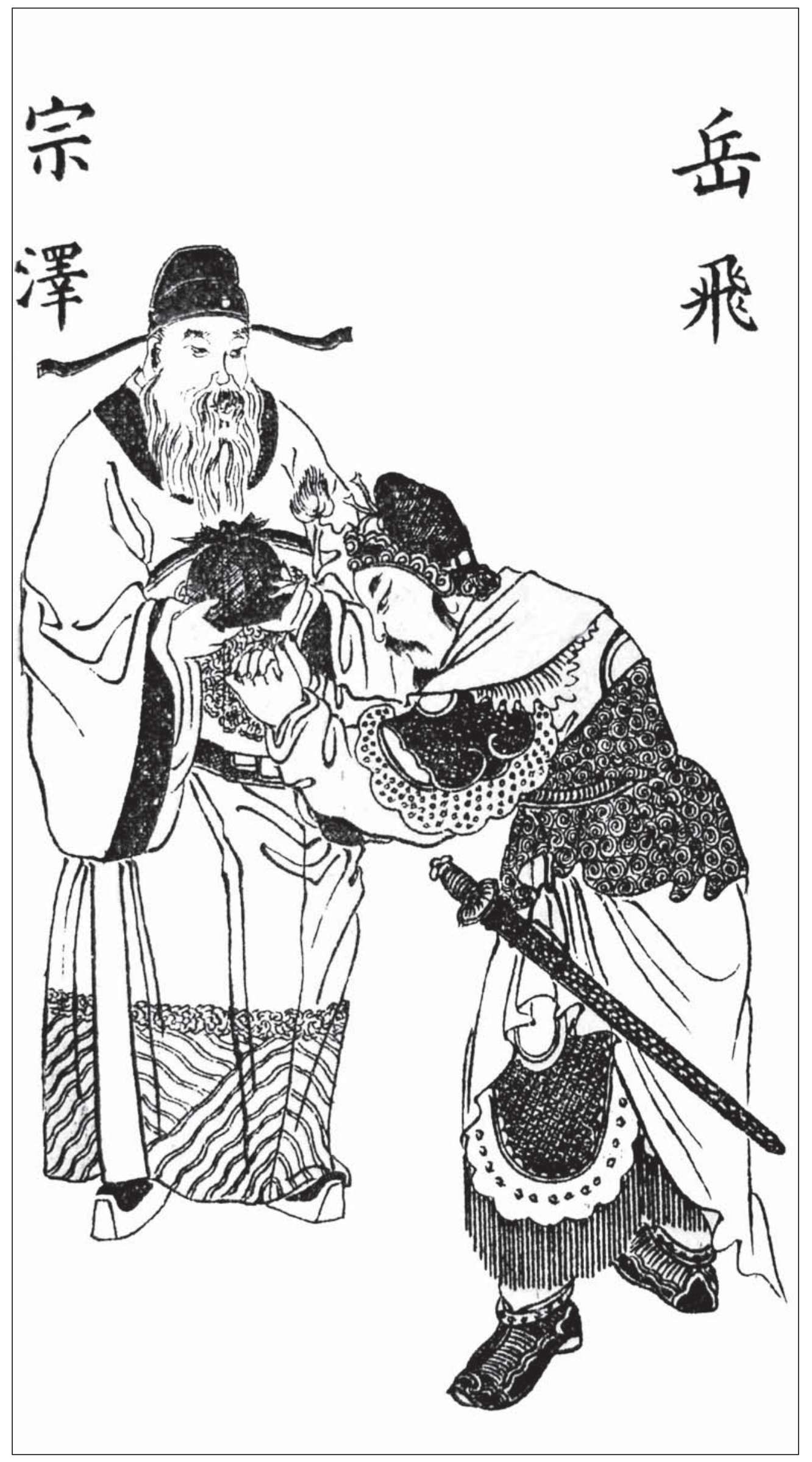




\title{
El general chino Yue Fei: hechos, relatos y misterios de las artes marciales
}

\author{
Stanley E. Henning
}

\begin{abstract}
Resumen
Yue Fei ( I I 03-1 I 42), el famoso general de la Dinastía Song del Sur que resistió las incursiones de los Jin (Yurchen) hasta que fue asesinado a la edad de 39 años a consecuencia de las intrigas de la corte, ha sido relacionado con un cierto número de escuelas de boxeo. A pesar de que los registros históricos no sustentan dicha relación, existieron razones para apoyar estos mitos, que han pervivido hasta el presente. Este artículo clarifica la conexión entre Yue Fei y las artes marciales a partir de la utilización de fuentes originales chinas, incluyendo el registro biográfico de Yue Fei y una visita al templo de Yue Fei en Hangzhou. Confirma las conocidas habilidades marciales de Yue Fei y explica los factores que subyacen a su reconocimiento como uno de los patriotas chinos más reverenciados y símbolo de las artes marciales.
\end{abstract}

Yue Fei (1103-1142), el famoso general de la Dinastía Song del Sur que resistió las incursiones de los Jin (Yurchen) hasta que fue asesinado a la edad de 39 años a consecuencia de las intrigas de la corte, ha sido relacionado con un cierto número de escuelas de boxeo. A pesar de que los registros históricos no sustentan dicha relación, existieron razones para apoyar estos mitos que han pervivido hasta el presente. Este artículo clarifica la conexión entre Yue Fei y las artes marciales.

Que Yue Fei estudió artes marciales parece indudable, tal y como se podría esperar de un líder militar de aquella época y tal y como registra adecuadamente su archivo genealógico. De acuerdo con el registro, Yue Fei empezó a estudiar técnicas de lanza a la edad de once años bajo la tutela de un profesor llamado Chen Guang, y se dice que llegó a ser tan hábil que era imbatible en su país en aquella época. Para cuando tenía trece años empezó a estudiar tiro con arco con Zhou Tong. Era extremadamente fuerte y podía tirar fácilmente de un arco de 300 jin o de una ballesta de ocho stones (sobre 300 libras en ambos casos [136 kg. aprox]). Cuando su profesor, Zhou Tong, murió, Yue Fei fue superado por la pena. Se vistió de duelo, se preparó para los sacrificios, disparó tres flechas de manera ceremoniosa para expresar sus respetos, y juró dedicarse a apoyar a su nación. Cuando llegó a ser general usó lo que había aprendido de Zhou Tong para entrenar a sus tropas, las cuales se hicieron famosas por su habilidad y disciplina.

Éstas son las únicas referencias registradas sobre el entrenamiento 
y práctica de las artes marciales por parte de Yue Fei. No se menciona el boxeo, aunque casi con toda seguridad practicase alguna forma de combate a manos desnudas como fundamento básico para el uso de armas.

Hoy día existen aproximadamente una docena de formas diferentes de boxeo esparcidas por nueve provincias que llevan el nombre de Yue Fei. La provincia de Hubei fue el escenario de las durísimas últimas batallas de Yue Fei contra las fuerzas Jin (a Yue se le concedió póstumamente el título de Príncipe de E [área de Wuchang, en la actual provincia de Hubei]). Es también el hogar de uno de los sistemas de boxeo Yue más concienzudamente organizados. Es posible que algo de este estilo haya evolucionado a partir de las formas existentes durante los tiempos de Yue Fei e incluso puede que estas formas fuesen practicadas por sus tropas, pero nada de esto ha podido verificarse históricamente.

Además de los estilos de boxeo que llevan el nombre de Yue Fei, una historia sobre los orígenes del boxeo Xingyi alega que éste fue creado por Yue Fei. Esta historia también asocia el boxeo Xingyi con la práctica de la lanza, una de las habilidades conocidas de Yue Fei. Por otro lado, la lanza fue, durante décadas, una de las habilidades marciales más ampliamente practicadas, y el boxeo Xingyi no es el único estilo en enfatizar la práctica de la lanza hoy en día. Como Chen Zongyou (1561-?), autor de la obra Elucidación de los métodos de bastón Shaolin señalaba, “... la lanza es el rey de las artes [marciales] ya que es el arma de la que resulta más difícil defenderse" (Cheng, 1621).

Como Douglas Wile ha apuntado en su Lost T'ai Chi Classics from the Late Ch'ing Dynasty (1996: 111), existe incluso una posibilidad de que el nombre del que se considera ahora el creador del Taijiquan, Wang Zongyue, fuese realmente una alusión patriótica a Yue Fei, ya que Zongyue significa "Reverenciar a Yue". De esta manera, podría ser más que una mera coincidencia que los que se han aceptado como los datos bibliográficos de Wang Zongyue vengan del Manual de Lanza Yinfu (siendo la lanza una de las famosas habilidades de Yue Fei), escrito por un individuo apellidado Wang y hallado por el historiador de las artes marciales Tang Dao (1897-1959) en los puestos de libros de Pekín en la década de 1930. En otras palabras, es posible que la así llamada Teoría del Taijiquan, descubierta en una tienda de

Entrada al templo de Yue Fei en la costa del agradable Lago Occidental, en Hangzhou, capital de la Dinastía Song del Sur (1127-1279).

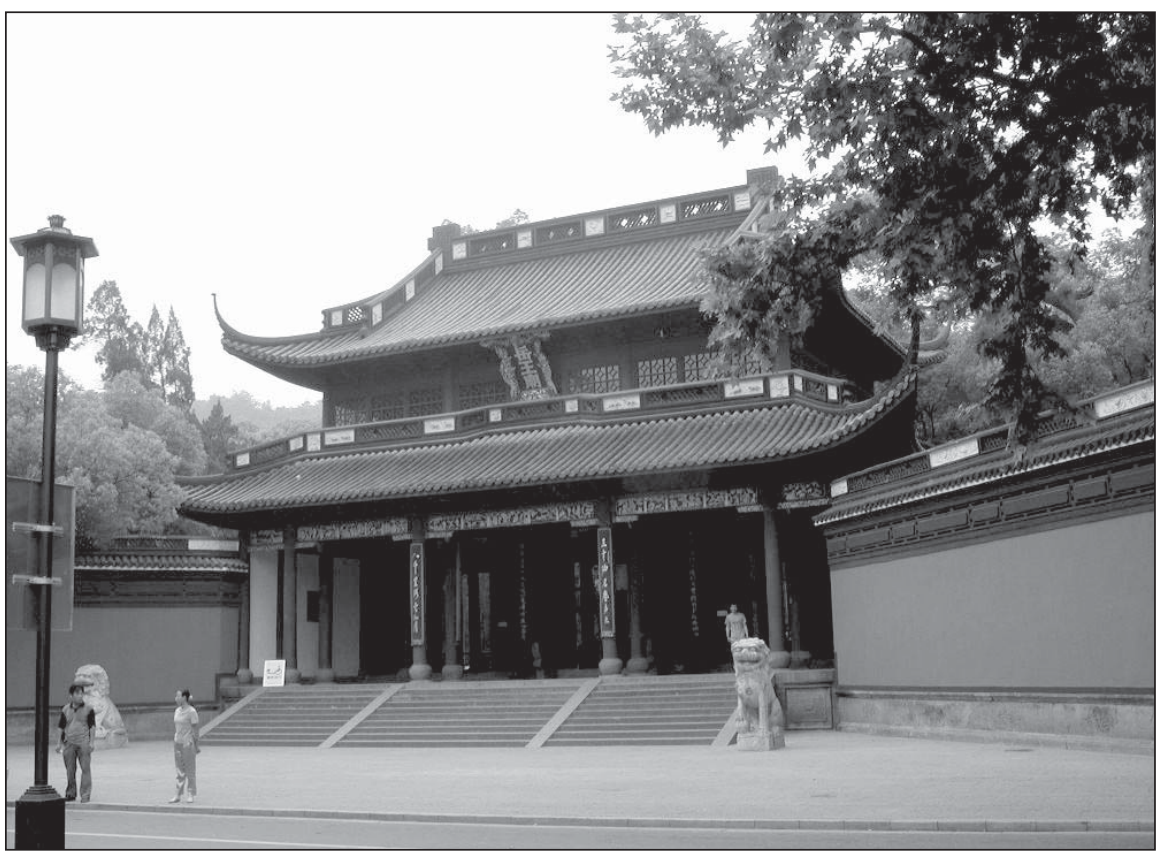




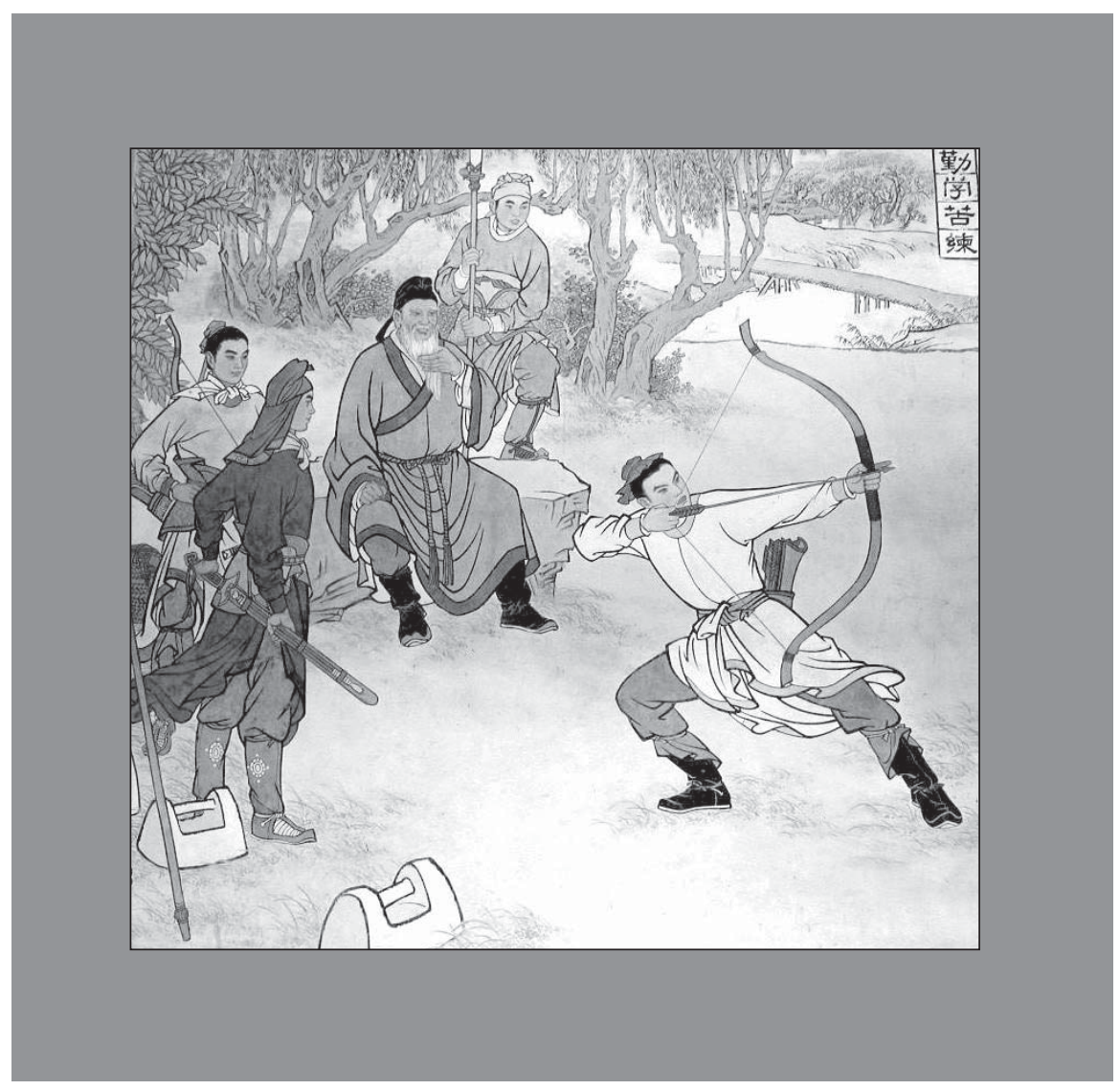

sal por el hermano del defensor del Taijiquan Wu Yuxiang a principios de la década de 1850 y atribuido a Wang Zongyue, fuese realmente un manual de lanza que inspiró a Wu a escribir Teoría del Taijiquan y a atribuirla a Wang Zongyue en honor de Yue Fei. Wu pudo también estar familiarizado con el Epitafio para Wang Zhengnan (1669) de Huang Zongxi, probablemente una crítica contra el gobierno Manchú, en el que uno de los llamados defensores de la Escuela Interna aparece como Wang Zong. De esta manera Wu, probablemente consciente de la relación de Yue Fei con la lanza, podría haber visto fácilmente esta potencial coincidencia con el nombre Wang Zong y una oportunidad ideal para continuar con el tema anti-Manchú de Huang a través de las artes marciales. De hecho, "Yinfu" se traduce como "Encantos secretos", y el Yinfu Jing (Clásico Yinfu) es un antiguo tratado militar que quizá dio nombre el manual de lanza. En cuanto al Manual de lanza Yinfu Tang Hao apunta que tiene semejanzas con la llamada Teoría del Taijiquan en sus referencias al yin y al yang, a la quietud y al movimiento, a la dureza y la suavidad, a lo sólido y lo vacío e incluso a la permanencia y el seguimiento (Tang, 1934b: 12).

El ejemplo de Yue Fei como líder tanto en la esfera civil (poesía y caligrafía) como militar (perspicacia en el combate), y su inquebrantable apoyo de la Dinastía Song del Sur contra las incursiones nómadas de los Jin lo convirtieron en el penúltimo símbolo del patriotismo chino (se dice que su madre le tatuó en su espalda cuatro caracteres correspondientes a "leal servicio al país"), y es lógico que estilos de artes marciales se denominasen después de él, especialmente como consecuencia de la conquista Manchú y su dominio sobre China desde 1644 a 1911.

Los Manchú eran descendientes de los fundadores Yurchen de la Dinastía Jin (1115-1234), contra los que luchó Yue Fei y, en 1616, Nuranchi (1559-1626), fundador del estado Manchú que finalmente conquistó China,
Mural en el templo de Yue Fei describiendo a Yue Fei estudiando tiro con arco bajo la tutela de su profesor, Zhou Tong. Fíjese en las pesas, conocidas como "piedras cerrojo", en la parte inferior izquierda.

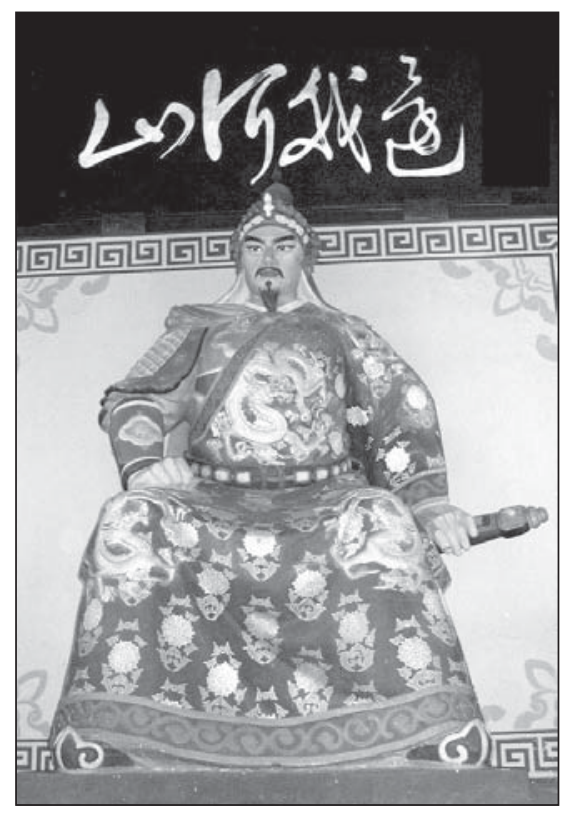

Estatua de Yue Fei en el templo de Yue Fei. La caligrafía en la mano de Yue Fei dice "devuelve nuestros ríos y montañas". 


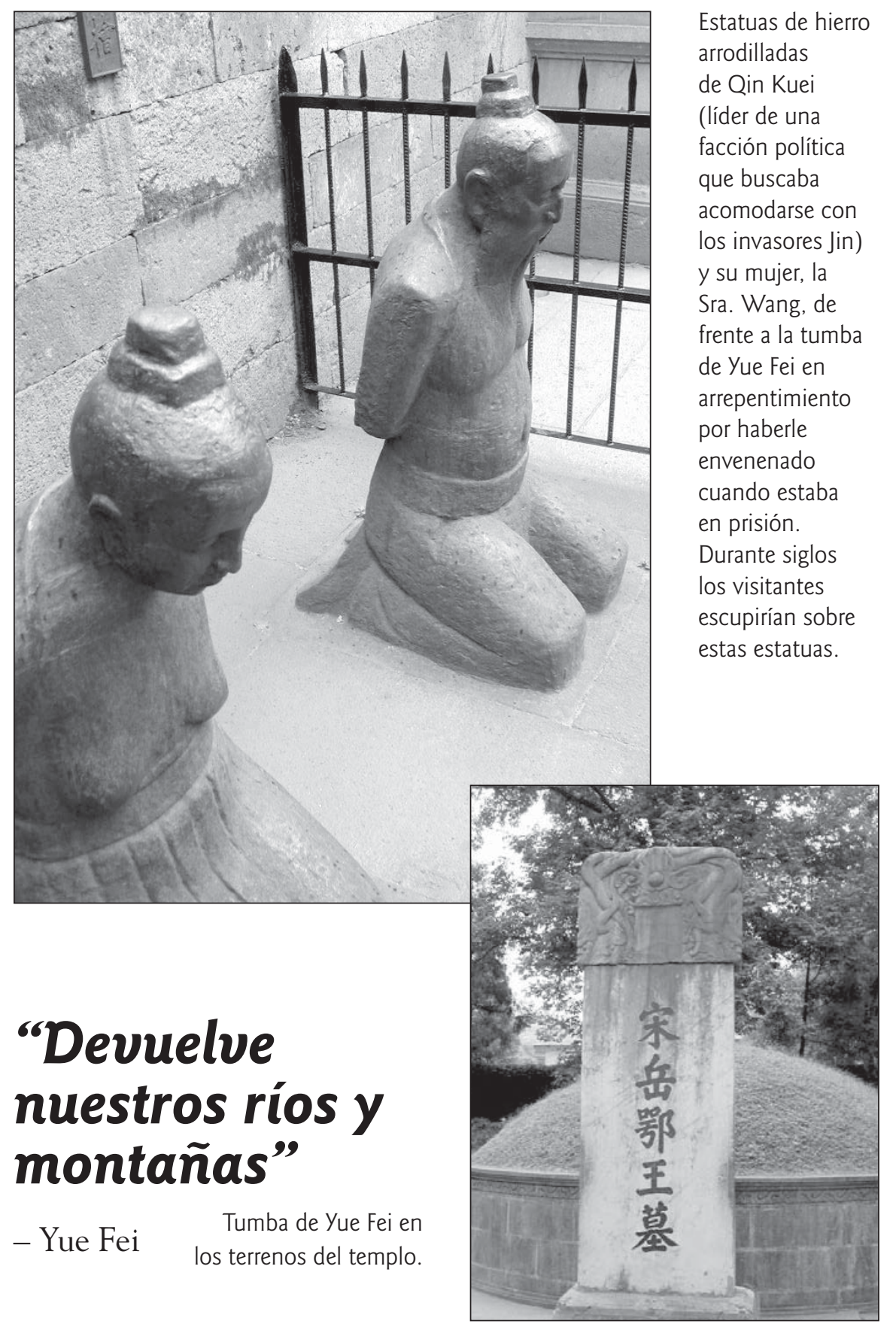

se llamó a sí mismo Emperador de la Última Dinastía Chin (Reischauer \& Fairbank, 1965: 352). De este modo, las artes marciales que llevan el nombre de Yue Fei podrían verse como símbolos de la resistencia china contra la dominación Manchú. Los gobernantes de la Dinastía Qing (1644-1912) eran sensibles a la simbología patriótica, y promulgaron un cierto número de decretos prohibiendo las prácticas de las artes marciales (que ellos llamaban Boxeo y Bastón). Durante el reinado del Emperador Qianglong (1736-1795) la novela popular Historia patriótica completa de Yue Fei fue puesta en una lista para destruirse.

El dominio Manchú sobre China finalizó hace casi cien años, pero los relatos sobre las conexiones de Yue Fei con las artes marciales subsisten como una evidencia del lazo entre el nacionalismo y patriotismo chino y su forma de cultura física más extendida. 


\section{Bibliografía}

\section{China}

中國武術協會, 審定 (1983). 中國武術拳械錄. 北京: 人民體育出版社.

錢彩 (1912). 繪圖精忠説岳全傳.上海:錦章圖書局.

錢汝雯, 編 (1924). 宋岳鄂王年譜.

唐豪(范生)(1935/1969a). 內家拳的研究. 香港:麒麟圖書公司.

唐豪(范生)(1935/1969b). 太極拳宗師王宗岳考. 香港:麒麟圖書公司.

王安寶, 魏峰 (編著) (2003). 心意六合拳發力與技擊. 北京:北京體育大學出版社.

無谷, 劉志學 (1984). 少林寺資料集續編. 北京:書目文獻出版社.

Chinese Martial Arts Association (1983). Zhongguo wushu quanxie lu [Registro de estilos de artes marciales chinas de boxeo y armas]. Beijing: People's Physical Culture Press.

Qian Cai (1912). Huitu shuoyue quan zhuan [Historia patriótica completa ilustrada de Yue Fei]. Shanghai: Jinzhang Press.

Qian Ruwen (1924). Song Yue E wang nianpu [Biografía de Song Yue, Príncipe de E (Wuchang, Hubei)]. Editor desconocido.

Tang Hao (Fansheng) (1935/1969a). Neijiaquan de yanjiu [Investigación sobre escuelas de boxeo internas]. Hong Kong: Unicorn Press.

Tang Hao (Fansheng) (1935/1969b). Taijiquan zongshi Wang Zongyue kao [Investigación sobre Wang Zongyue, creador del Taijiquan]. Hong Kong: Unicorn Press.

Wang Anbao, \& Wei Feng (Eds.). (2003). Xinyi Liuhequan fali yuJiji [El combate y la liberación de fuerza del Boxeo de las Seis Combinaciones Xinyi]. Beijing: Tiyu Daxue Press.

Wu Gu \& Liu Zhixue (Eds.). (1984). Shaolinsi ziliaoji xubian [Continuación de la colección de materiales de referencia del Monasterio Shaolin]. Beijing: Shumu Wenxian Press.

\section{Inglesa}

Goodrich, L. (1935). The literary inquisition of Ch'ien-Lung. Baltimore: Waverly Press.

Reischauer, E., \& Fairbank, J. (1965). East Asia: The great tradition. Tokyo: Charles Tuttle. Modern Asia Edition.

Wile, D. (1996). Lost T'ai Chi classics from the late Ch'ing dynasty. Albany: State University of New York Press.

\section{Glosario}

\begin{tabular}{|c|c|c|}
\hline Pinyin & Español & Chino \\
\hline $\begin{array}{l}\text { Chen Guang } \\
\text { quanbang }\end{array}$ & Boxeo & $\begin{array}{l}\text { 棵廣 } \\
\text { 拳棒 }\end{array}$ \\
\hline Jin Chao & Dinasatía Jin & 金朝 \\
\hline jinzhong baoguo & Leal servicio al país & 盡忠報國 \\
\hline $\begin{array}{l}\text { Yue E Wang } \\
\text { Qin Kui }\end{array}$ & Príncipe Yue de E & $\begin{array}{l}\text { 岳鄂王 } \\
\text { 秦檜 }\end{array}$ \\
\hline Qing Chao & Dinastía Qing & 清朝 \\
\hline huan wo hezhan & Devuelve nuestros ríos y montañas & 還我河山 \\
\hline Nan Song & Song del Sur & 南宋 \\
\hline shisuo & Piedras cerrojo & 石鎖 \\
\hline Wang Zongyue & & \\
\hline $\begin{array}{l}\text { Yinfu Qiangpu } \\
\text { Yue Fei } \\
\text { Zhou Tong }\end{array}$ & Manual de lanza Yinfu & $\begin{array}{l}\text { 陰符槍譜 } \\
\text { 岳飛 } \\
\text { 周同 }\end{array}$ \\
\hline
\end{tabular}

